\title{
Space Qualification of the Optical Filter Assemblies for the ICESat-2/ATLAS Instrument
}

\author{
E. Troupaki*a ${ }^{* a}$, Z. H. Denny ${ }^{b}$, S. Wu ${ }^{a}$, H. N. Bradshaw ${ }^{a}$, K. A. Smith ${ }^{\mathrm{c}}$, J. A. Hults ${ }^{\mathrm{c}}$, L. A. Ramos- \\ Izquierdo ${ }^{\mathrm{a}}, \mathrm{W} . \mathrm{B}$. Cook $^{\mathrm{a}}$ \\ ${ }^{\mathrm{a}}$ NASA Goddard Space Flight Center, Greenbelt, MD; ${ }^{\mathrm{b} S S A I}$ Inc., Lanham, MD; ${ }^{\mathrm{c} O r b i t a l}$ Sciences \\ Corp., Dulles, VA
}

\begin{abstract}
The Advanced Topographic Laser Altimeter System (ATLAS) will be the only instrument on the Ice, Cloud, and Land Elevation Satellite -2 (ICESat-2). ICESat-2 is the 2nd-generation of the orbiting laser altimeter ICESat, which will continue polar ice topography measurements with improved precision laser-ranging techniques. In contrast to the original ICESat design, ICESat-2 will use a micro-pulse, multi-beam approach that provides dense cross-track sampling to help scientists determine a surface's slope with each pass of the satellite. The ATLAS laser will emit visible, green laser pulses at a wavelength of $532 \mathrm{~nm}$ and a rate of $10 \mathrm{kHz}$ and will be split into 6 beams. A set of six identical, thermally tuned optical filter assemblies (OFA) will be used to remove background solar radiation from the collected signal while transmitting the laser light to the detectors. A seventh assembly will be used to monitor the laser center wavelength during the mission. In this paper, we present the design and optical performance measurements of the ATLAS OFA in air and in vacuum prior to their integration on the ATLAS instrument.
\end{abstract}

Keywords: ATLAS, ICESat-2, etalon, receiver, solar background

\section{INTRODUCTION}

ATLAS is the sole instrument on the ICESat-2 observatory. The ICESat-2 mission is a follow- on to the ICESat mission and is being targeted for launch in 2017. ATLAS has been designed to collect altimetry measurements of the Earth's surface, optimized to measure the heights and freeboard of polar ice and generate an estimate of global vegetation biomass ${ }^{1,2}$. The mission life is three years plus 60 days of commissioning. The ATLAS laser transmitter can generate over $9 \mathrm{~W}$ at $532 \mathrm{nn}$ with a frequency of $10 \mathrm{KHz}$ and less than $1.5 \mathrm{~ns}$ pulsewidth $(\mathrm{FWHM})^{3}$. It is frequency tunable and is split into 6 beams. ATLAS is a photon-counting laser altimeter, which means it can measure distance by detecting just a few photons from each laser pulse and timing their round-trip travel from satellite to earth and back very accurately.

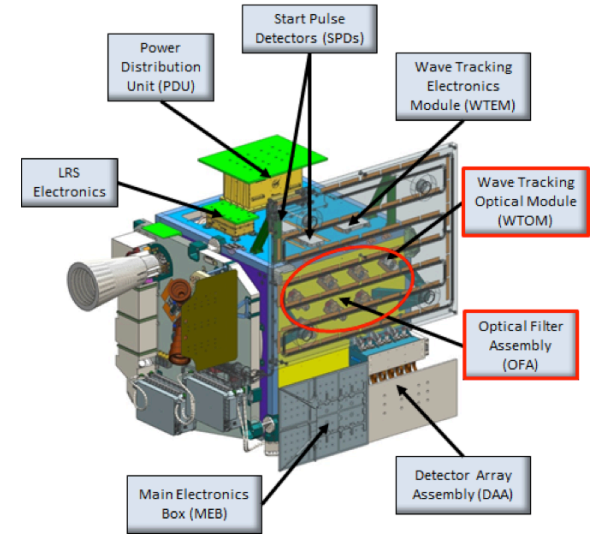

Figure 1: ATLAS receiver subsystems

\footnotetext{
* elisavet.troupaki-1@nasa.gov, (301) 614-6119
} 
The ATLAS Optical Filter Assembly (OFA) is required to remove background solar irradiation from the collected laser signal while transmitting the laser light to the ATLAS detectors and also to provide the means to monitor the etalon tuning to the laser wavelength (wavelength tracking). The schematic in figure 1 shows the current position of the OFA and other receiver subsystems on the ATLAS instrument.

At the OFA, each fiber optic carrying a laser spot from the ATLAS Receiver Telescope Assembly (RTA) goes through an etalon optical filtering module (OFM). The etalons provide an optical band-pass filter with a thermally-controllable center wavelength nominally set at the laser center wavelength of $532.272 \mathrm{~nm}$ (in vacuum) and a very narrow bandwidth. Each of the six laser spots that pass through the OFA are transmitted to their own photomultiplier tube (PMT) in the Detector Array Assembly (DAA).

In addition to the six OFM, there is a seventh etalon module, called the Wavelength Tracking Optical Module (WTOM), that receives a sample of the laser pulse and monitors the laser center wavelength using a technique similar to the one on ICESat-1. Two laser light beams are being transmitted through the WTOM etalon; one beam is normal to the etalon and the second at a smaller angle. The two beams are being measured by the detectors in the Wavelength Tracking Electronics (WTEM) box.

\section{DESIGN AND TRADE STUDIES}

The ICESat-2 mission requirements evolved substantially after the initial design for the receiver assembly was started. The use of optical fibers to collect and control the input greatly relaxed constraints on component placement within the instrument/spacecraft. Initially 16 beams were planned, but changes in mission requirements soon reduced this to 9 and eventually to the actual 6 beams to be employed. The fiber connectors to be used forced a relatively large separation between individual beams at both the focal plane of the telescope and at the front of the originally planned single etalon filter. Thermal and mechanical analyses showed that using a reasonable mechanical design temperature gradients across the etalon would likely not be controlled well enough to meet the signal uniformity requirement (no more than $5 \%$ difference between highest and lowest beam signal magnitudes). The solution we chose was to employ 6 separate thermally-tuned etalon assemblies, one for each beam, that could be tuned individually to establish and maintain optimal transmission of the received laser signals.

Because ATLAS measures laser returns at a single stable wavelength, there is no need for broadband tuning of the etalon filters so a solid etalon's mechanical advantages made using a solid etalon the best choice for ATLAS. The major disadvantage of using solid etalons for ATLAS is in optimizing the etalon transmission since the thermal tuning range is much more restricted than that of an etalon with individually controlled plates. This optimization is provided to first order by manufacturing the etalon to match thickness and surface quality specifications very precisely, and then fine tuning using moderate thermal changes. The material chosen for the ATLAS solid etalons is fused silica. For a wavelength near $532 \mathrm{~nm}$, a free spectral range (FSR) of $300 \mathrm{pm}$ and a full width at half maximum (FWHM) of $30 \mathrm{pm}$, the etalon needs to operate at or near order 1775 . This in combination with the refractive index $\left(\sim 1.461\right.$ at $\left.22^{\circ} \mathrm{C}\right)$ and thermal expansion coefficients for fused silica set the etalon thickness to be approximately $0.032 \mathrm{~cm}$. An etalon this thin is quite fragile, and so a "sandwich" arrangement using two thicker plates of fused silica on either side of the etalon surfaces was used to provide sufficient mechanical strength. The etalon surfaces were coated to provide the correct reflectivity to achieve the required FSR and FWHM (R 93\%), and the outer surfaces were anti-reflection coated (R< $0.1 \%$ at $532 \mathrm{~nm})$.

During development the final laser wavelength was not well defined, nor was the manufactured etalon thickness yet known, so the thermal control system was designed to allow for at least $+/-16 \mathrm{pm}$ shift from a nominal operating value eventually chosen to be $40^{\circ} \mathrm{C}$. The laser specifications were eventually changed to provide for tuning of the laser wavelength, eliminating the need to tune the etalons to match it (though the etalons must still be tuned to match each other). The manufactured etalons were very close to the nominal value specified for them. Hence there is significant margin in the thermal control system beyond that needed to optimize transmission.

The thermal stability of the optical properties of fused silica is very good, and this is an advantage in general since maintaining the etalon's peak transmission alignment with the laser wavelength does not require frequent monitoring and adjustment The ATLAS OFA thermal system is designed to maintain temperatures to better than $0.2^{\circ} \mathrm{C}$ at the operating temperature which keeps the transmission stable to within $2 \%$ of the optimized value. Thermal control is provided for 
the entire OFA assembly as a unit and not just for the etalons. This provides a larger mass to help maintain stability while still allowing temperature tuning.

Tuning the etalons via tilting them slightly is not a good option for ATLAS and was not considered since it would lead to unacceptable performance loss. Analyses of the potential distributions across the filter input aperture showed that for the range of expected bore sight drift allowed by requirements on other ATLAS system components the change in filter transmission could become unacceptably low for even very small $(<10)$ tilts in the etalon. Contrast is also significantly affected by tilting the ATLAS etalons and would have led to some increase in the amount of background (reflected solar) light allowed through the etalons.

A blocking ("coarse") filter is used to eliminate solar background transmitted through the orders of the ATLAS etalons other than the one aligned with the laser wavelength. This filter's spectral FWHM must be smaller than the etalon FSR and preferably much smaller so that the "wings" of the blocking filter's spectral transmission function are very small at the spectral locations of the etalon's nearest side orders. The blocking filter should must also have a high peak transmission at the laser wavelength and peak of the etalon transmission function. The design specifications for the ATLAS blocking filters are FWHM of $200 \mathrm{pm}$ and a peak transmission $>80 \%$. Other considerations in blocking filter performance that are more difficult to specify or validate include rapid roll-off of the transmission away from the peak and the level of total out-of-band blocking. For the design, a Gaussian shape was assumed for the blocking filter's spectral transmission up to 4 FWHM from the peak and a general optical density (OD) of 5 was specified for the rest of the spectrum within the sensing range of the detectors $(250-950 \mathrm{~nm})$. Given the specification on the etalon and filters, out-of-band transmission was expected to be $<9 \mathrm{e}-03 \%$.

The optical arrangement for the etalon/blocking filter system is straightforward, a collimating lens collects the output from the optical fiber, directs the light through the filters, and the filtered light is collected by a focusing lens and sent into an over-sized optical fiber for delivery to the detector sub-assembly. Lens, etalon and blocking filter diameters were set to be slightly larger than required to meet performance requirements derived by considering the fiber aperture size, restrictions on aperture effects, focal length of the lenses (and effects on overall size of the OFA), and the need to include a means to tap into the optical system for other tests. The etalons are small and thus relatively easily made with the desired performance, and they could have been made larger if necessary. The lens focal lengths were kept as small as possible while retaining acceptable performance of the system. The ATLAS instrument calibration and validation requires the ability to sample and introduce test laser signals and the OFA was found to be the best place to insert these test points. Hence beamsplitters and additional focusing lenses are incorporated into the OFA. These are used to direct the light to or from optical fiber-fed apertures and eventually connected to external test components (see figure 2 ).

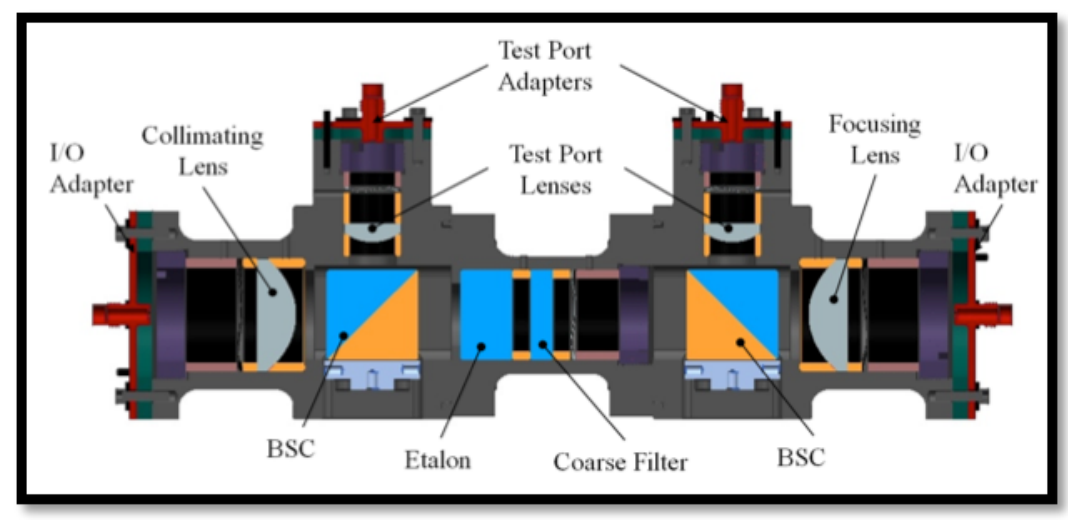

Figure 2: cross section of one the ATLAS Optical Filtering Module (OFM)

In the WTOM, we use a 2-channel DOE (Diffractive Optical Element) beamsplitter and an etalon as well as focusing and collimating lenses. Two laser light beams are being transmitted through the etalon; one beam is normal to it (onaxis) and the second at a smaller angle (off-axis) so that their relative transmission through the etalon (On-axis/Off-axis) is higher than 1. This allows for shifts in laser wavelength to be measured by the two wavelength tracking detectors due to the change in a power ratio between on-axis and off-axis beams through the etalon filter. The center wavelength of the WTOM's etalon filter is assumed to be constant, as it will be kept at a constant temperature by integrated heaters. If the laser drifts up or down in wavelength, the on-axis and off-axis channels will behave in distinct ways that can be measured and used to indicate the change in peak laser wavelength. If the on-axis to off-axis ratio decreases, then the 
laser's wavelength has drifted to shorter wavelengths; whereas if the ratio increases the wavelength has drifted to longer wavelengths. If the wavelength shift occurs during normal operation of one of the onboard lasers, the wavelength tracking electronics will signal the laser to tune its wavelength back to the center wavelength of the OFA etalon filters.

\section{ASSEMBLY AND OPTICAL PERFORMANCE}

In summary, the design of the ATLAS OFA subsystem was driven by the following requirements:

- The OFA shall have an optical pass band having the shape of a single cavity etalon with full width at half maximum of $30 \pm 3 \mathrm{pm}$.

- The OFA shall have a tuning range greater than $\pm 15 \mathrm{pm}$ centered on the nominal laser wavelength of $532.272 \mathrm{~nm}$ (vacuum).

- The total transmittance of the OFA, integrated across the solar spectrum shall be less than $8.5 \mathrm{E}-3 \%$.

- The OFA shall have total optical throughput values greater than or equal to $64.4 \%$.

- The OFA shall provide a means to monitor the relative wavelength tuning between itself and the ATLAS laser.

The etalon filters were manufactured by Lightmachinery Inc. and the coarse filters by Materion Barr. A sample coarse filter was sent to the etalon manufacturer for a cross-check of the center wavelenth before delivery. In parallel, the OFA lab wavemeter was cross-calibrated with the ATLAS laser wavemeter used by Fibertek (ATLAS laser manufacturer) at their facility. The 2-channel DOE beamsplitter was manufactured by Jenoptik. All OFA optics are made of fused silica.

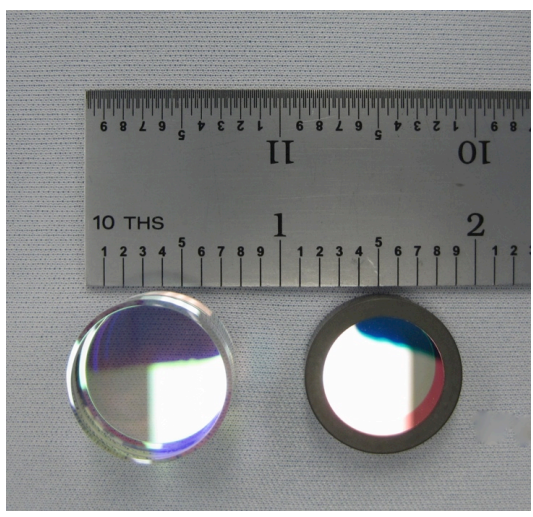

Figure 3: ATLAS etalon (left) and interference (coarse) filter (right).

Tables 1, 2 and 3 below show the measured performance of the filters and DOE measured in the ATLAS OFA lab. All optics met and often exceeded specifications. The filters were tested at different temperatures using a thermally controlled mount and measured with a low-power, single-mode frequency-doubled tunable 532nm lab laser. We bought 18 etalons and 20 filters and selected the flight parts and flight spares for the six OFM units in etalon-filter pairs so that their center wavelength would match each other best at the mission operating temperature. We bought four DOE beamsplitters and selected one for flight. The DOE transmission was measured with a green laser and the diffraction angle measurements with a CCD camera and a theodolite.

Table 1: Measured performance of the 18 ATLAS etalon filters

\begin{tabular}{|c|c|c|}
\hline Parameter & Specification & Performance \\
\hline Center Wavelength & $\begin{array}{c}532.272 \mathrm{~nm}+/-0.012 \mathrm{~nm} \\
\text { in vacuum, at } 40^{\circ} \mathrm{C}\end{array}$ & $532.264 \mathrm{~nm}-532.283 \mathrm{~nm}$ \\
\hline Free Spectral Range & $300+/-3 \mathrm{pm}$ & $297.6 \mathrm{pm}-299.9 \mathrm{pm}$ \\
\hline Passband full width & $\begin{array}{c}30+/-2 \mathrm{pm} \text { at } 50 \% \text { of measured peak } \\
\text { transmission }\end{array}$ & $27.73 \mathrm{pm}-28.80 \mathrm{pm}$ \\
\hline Peak transmission & $>90 \%$ & $96.01 \%-98.20 \%$ \\
\hline
\end{tabular}


Table 2: Measured performance of the 20 ATLAS interference (coarse) filters

\begin{tabular}{|c|c|c|}
\hline Parameter & Specification & Performance \\
\hline Center Wavelength & $\begin{array}{c}532.272 \mathrm{~nm}+/-0.040 \mathrm{~nm}, \\
\text { at } 40^{\circ} \mathrm{C}, \text { in vacuum }\end{array}$ & $532.245 \mathrm{~nm}-532.285 \mathrm{~nm}$ \\
\hline FWHM & $200+/-40 \mathrm{pm}$ & $184.0 \mathrm{pm}-195.7 \mathrm{pm}$ \\
\hline Peak transmission & $>80 \%$ & $82.2 \%-90.3 \%$ \\
\hline Out-of-band blocking & OD5 from $250 \mathrm{~nm}$ to $950 \mathrm{~nm}$ & OD5 or better \\
\hline
\end{tabular}

Table 3: Measured performance of the 4 WTOM DOE beamsplitters

\begin{tabular}{|l|l|l|}
\hline Parameter & Specification & Performance \\
\hline $1^{\text {st }}$ order deviation & $7.3 \mathrm{mrad}$ & $7.26 \mathrm{mrad}-7.31 \mathrm{mrad}$ \\
\hline Transmission efficiency & $\begin{array}{l}>73 \% \text { input beam energy in two } \\
\text { diffracted beams }\end{array}$ & $89.4 \%-91.9 \%$ \\
\hline
\end{tabular}

Table 4 below summarizes the optical performance of the six assembled OFM units. The optical transmission reported here was measured with the same single-mode narrow-band tunable green laser that we used previously to measure the transmission of the individual filters. In addition to that, we measured the optical transmission of the OFM with one of the prototype ATLAS lasers, currently under lifetest at Goddard Space Flight Center (GSFC) and the difference (drop) did not exceed $5 \%$ of what we measured in the OFA lab.

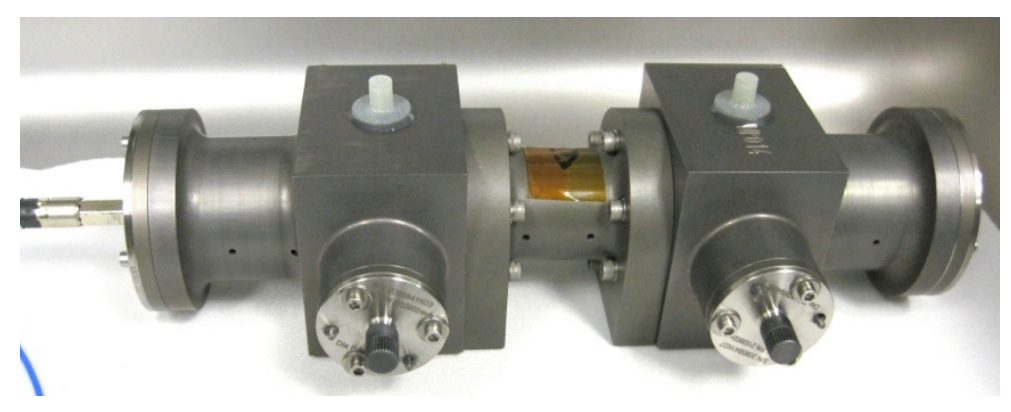

Figure 4: One of the 6 assembled ATLAS OFM.

Table 4: Optical performance of the 6 fligth OFM

\begin{tabular}{|l|l|l|}
\hline \multicolumn{1}{|c|}{ Parameter } & \multicolumn{1}{|c|}{ Requirement } & \multicolumn{1}{c|}{ Performance } \\
\hline Optical transmission & $64.4 \%$ & $\begin{array}{l}71.2 \%-78.7 \% \text { (lab single-mode } \\
\text { tunable 532nm laser); }\end{array}$ \\
\hline Bandwidth (FWHM) & $30 \pm 3 \mathrm{pm}$ & $31.04-32.35 \mathrm{pm}$ \\
\hline Center wavelength (vacuum) & $532.272 \mathrm{~nm}$ & $\begin{array}{l}532.272 \mathrm{~nm} \text {, at nominal operating } \\
\text { temperature }\end{array}$ \\
\hline Wavelength tunability & greater than $\pm 15 \mathrm{pm}$ & greater than 25pm \\
\hline Solar background rejection & $\begin{array}{l}\text { transmittance of no more than 8.5E- } \\
5 \% \text { integrated across the solar } \\
\text { spectrum }\end{array}$ & $\begin{array}{l}3.7 \mathrm{E}-5 \% \text { - 8.5E-5\% broadband } \\
\text { transmission between 250nm and } \\
950 \mathrm{~nm}\end{array}$ \\
\hline Light tightness & $\begin{array}{l}\text { Stray light events of no more than } \\
\text { 2KHz light }\end{array}$ & $<500 \mathrm{~Hz}$ (lab) \\
\hline
\end{tabular}




\section{ENVIRONMENTAL TESTING}

Space qualification testing of the six flight OFM and one WTOM units was performed after assembly and baseline performance testing in the lab and included vibration testing and thermal-vacuum (TVAC) testing. . One engineering OFM unit had been previously tested at higher levels (GEVS) with no major issues.

\subsection{Vibration Testing}

We performed protoflight level sine vibration, random vibration and sine burst testing on all 6 flight OFM and the WTOM in order verify that the OFA subassembly is capable of withstanding launch without degradation. Table 5 below summarizes the test levels for sine burst and swept sine vibration and figure 5 shows the test levels for random vibration.

Table 5: Test levels for sine burst and swept sine vibration tests

\begin{tabular}{|c|c|c|}
\hline Type of testing & Axis & Protoflight Level \\
\hline Sine Burst & $\mathrm{X}, \mathrm{Y}, \mathrm{Z}$ & $15 \mathrm{G}, 5$ cycles conducted at $25 \mathrm{~Hz}$ \\
\hline Swept Sine Vibration & $\mathrm{X}, \mathrm{Y}, \mathrm{Z}$ & 0.63 inches D.A. (5-19.7Hz, 4 oct/min sweep rate) \\
& & $12.5 \mathrm{G}(19.7-50 \mathrm{~Hz}, 4 \mathrm{oct} / \mathrm{min}$ sweep rate) \\
\hline
\end{tabular}
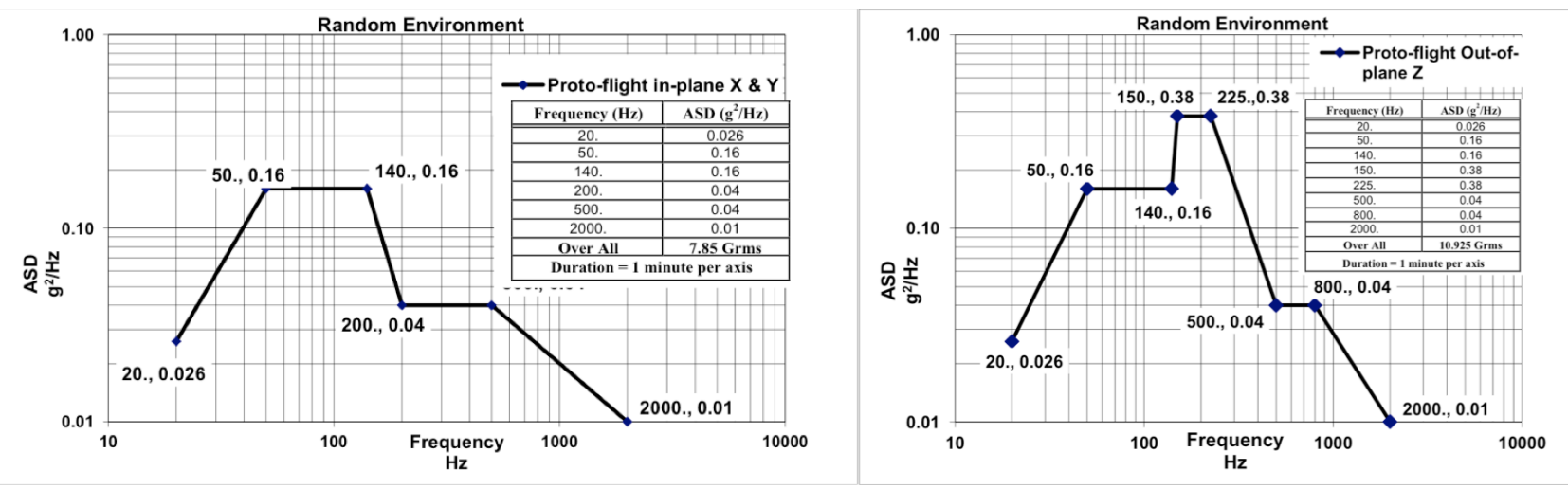

Figure 5: Random vibration levels for $\mathrm{x}$ and $\mathrm{y}$ axis (left) and $\mathrm{z}$ axis (right)

The variances between the pre and post vibration sine sweeps peak frequencies for each axis were within the tolerance to conclude that the structural integrity of all OFMs was maintained during vibration testing. Pre and post vibration optical performance was within our measurement repeatability.

\subsection{Thermal-Vacuum Testing}

A Thermal-vacuum test was also performed on all optical modules after the vibration test and demonstrated hardware integrity, including the proper functioning of the flight thermistors, thermostats, and heater. The units endured one survival cycle in vacuum between $-20^{\circ} \mathrm{C}$ and $65^{\circ} \mathrm{C}$ and three qualification cycles in-air between $25^{\circ} \mathrm{C}$ and $55^{\circ} \mathrm{C}$. A bakeout was also performed during the test to ensure cleanliness of the hardware before integration at higher levels of assembly (see figure 7 for complete TVAC test profile). One OFM was illuminated with a green beam set at the ATLAS center laser wavelength of $532.272 \mathrm{~nm}$ during the test and its transmission was continuously monitored over time and temperature (figure 8). As expected, optical transmission reached peak values as the temperature of each OFM reached its nominal value. All values of optical throughput were meeting the mission requirement of at least $64.4 \%$ in vacuum. 


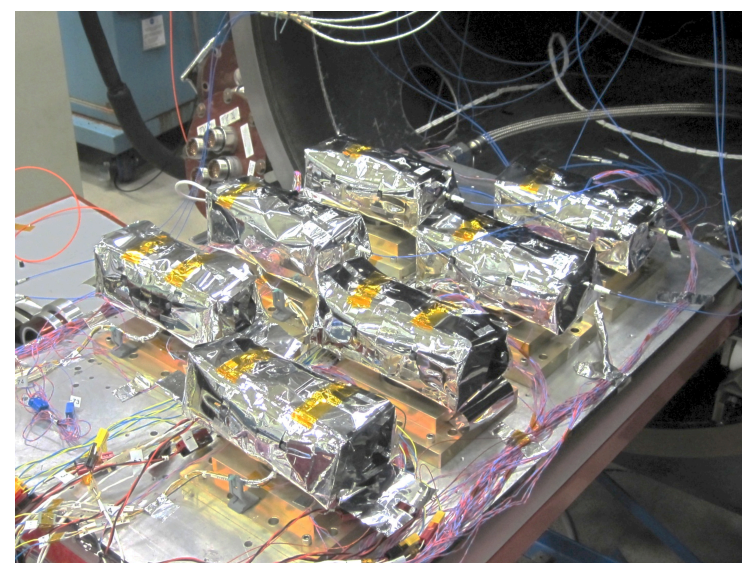

Figure 6: The OFA units - in thermal blankets - entering the vacuum chamber

This was the first time the flight units operated in vacuum at the projected mission operating temperatures and therefore their optical transmission and peak wavelength requirements were verified. A transmission check was also performed in air at the same operating temperature while in the chamber and no apparent effect of the vacuum conditions on the optical performance was observed.

To verify the OFM is tunable, a laser wavelength versus optical transmission measurement was performed at a temperature below and above nominal OFM temperature. Predictable wavelength-shifted optical transmission peaks were observed for each respective measurement and temperature coefficients of $\sim 3.6 \mathrm{pm} /{ }^{\circ} \mathrm{C}$ were measured (figure 9). Optical transmission and center wavelength measurements were repeated in the lab after the TVAC test and results were in good agreement with pre-test data (see table 6).

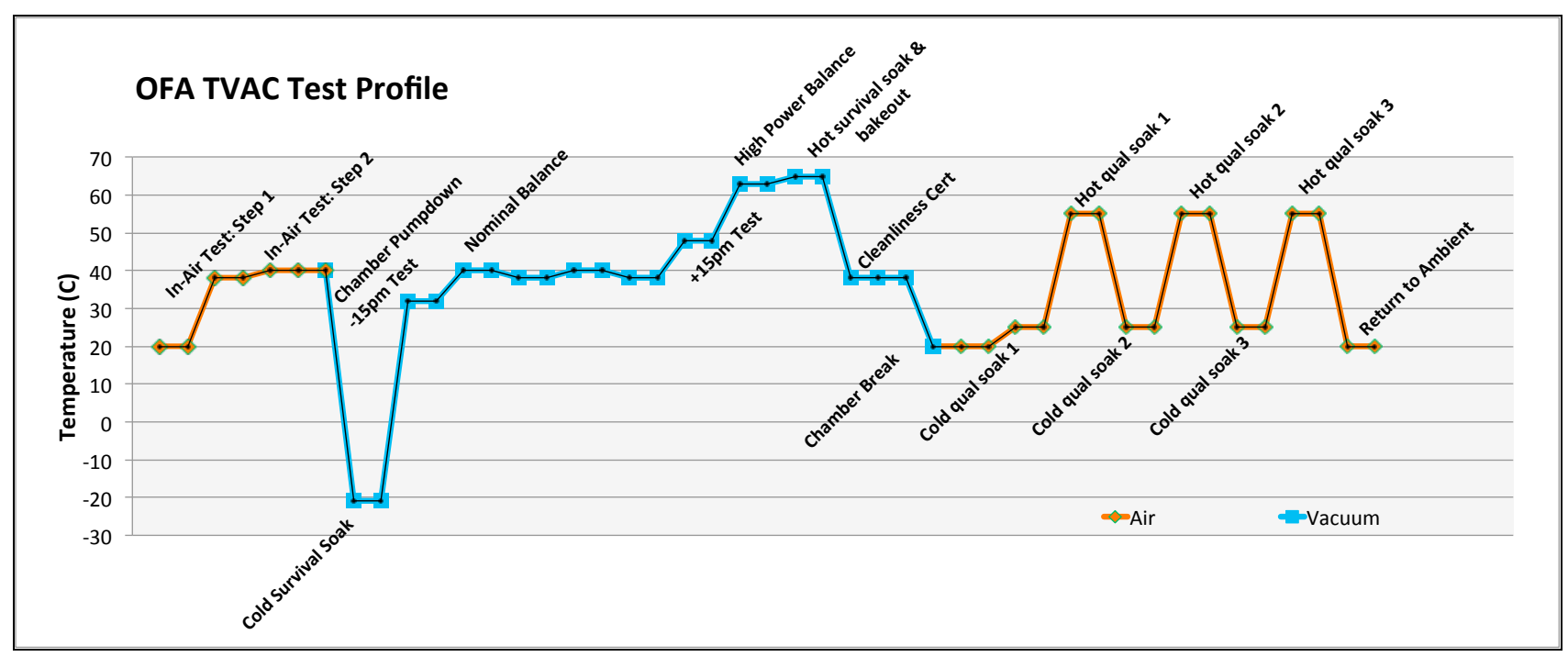

Figure 7: OFA TVAC test profile 


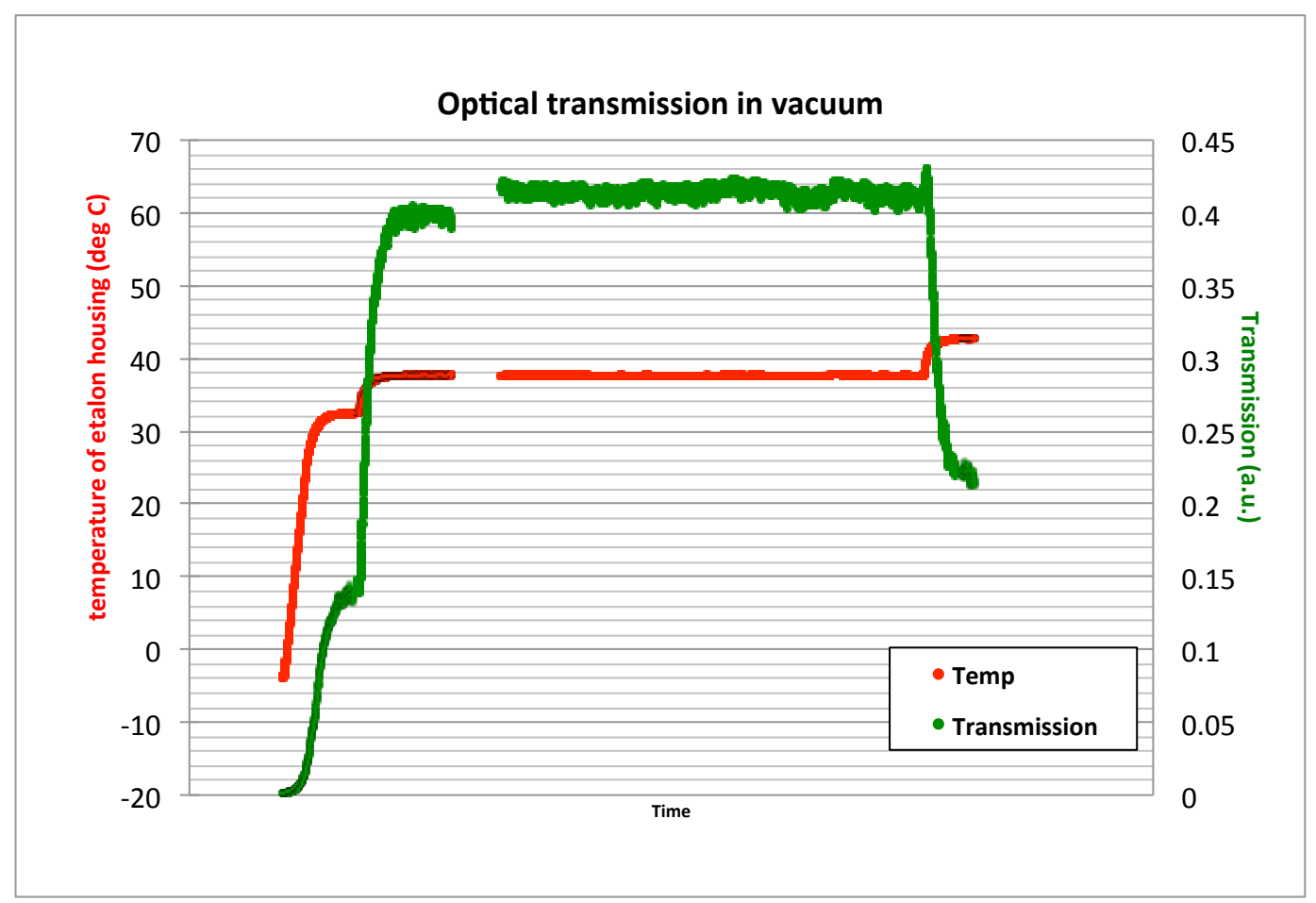

Figure 8: Optical transmission and temperature of te etalon housing of one OFM during TVAC test as a function of time

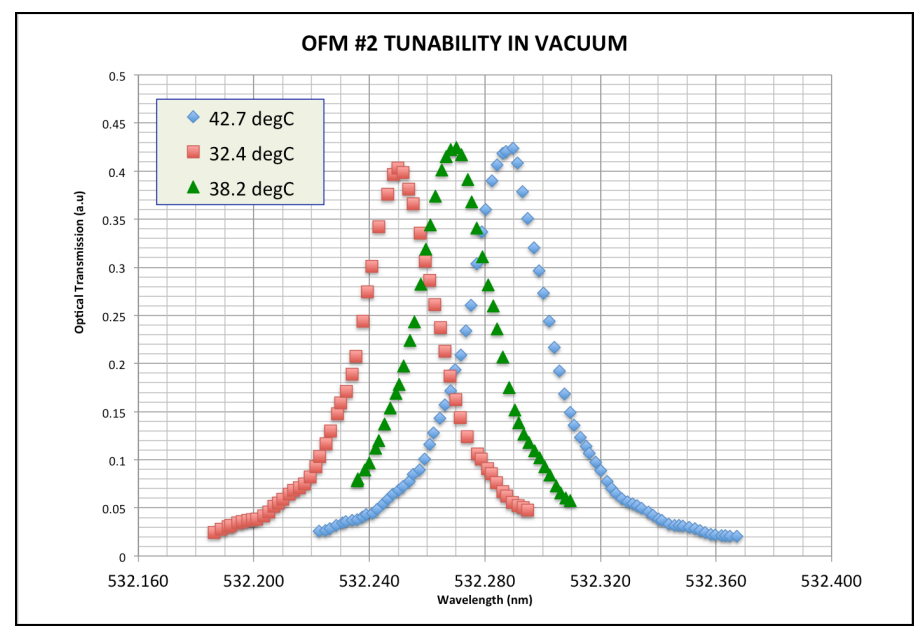

Figure 9: Tunability test of one OFM in vacuum: temperature coefficient measured to be $\sim 3.6 \mathrm{pm} /{ }^{\circ} \mathrm{C}$.

Table 5: Center wavelength data before and after vibration and vacuum testing

\begin{tabular}{|l|c|c|c|c|}
\hline \hline \multicolumn{2}{|c|}{} & \multicolumn{3}{|c|}{ Center Wavelength at Nominal Temperature (nm, vacuum) } \\
\hline OFM \# & $\begin{array}{c}\text { Nominal Temp } \\
\left({ }^{\circ} \mathrm{C}\right)\end{array}$ & baseline test & after vibration & after TVAC \\
\hline 1 & 40.71 & 532.272 & 532.272 & 532.270 \\
\hline 2 & 38.74 & 532.272 & 532.272 & 532.272 \\
\hline 3 & 37.25 & 532.272 & 532.273 & 532.273 \\
\hline 4 & 41.44 & 532.272 & 532.270 & 532.270 \\
\hline 5 & 41.52 & 532.272 & 532.275 & 532.274 \\
\hline 6 & 42.05 & 532.272 & 532.276 & 532.271 \\
\hline $\begin{array}{l}\text { WTOM } \\
\text { CH1 }\end{array}$ & 39.40 & 532.272 & 532.271 & 532.273 \\
\hline $\begin{array}{l}\text { WTOM } \\
\text { CH2 }\end{array}$ & 39.40 & 532.266 & 532.268 & 532.266 \\
\hline
\end{tabular}




\section{CONCLUSION}

The ATLAS Optical Filter Assembly (OFA) has been designed to remove background solar irradiation from the collected laser signal while transmitting the laser light to the ATLAS detectors and also to provide the means to monitor the etalon tuning to the laser wavelength (wavelength tracking). It consists of six identical etalon filter assemblies and one wavelength tracking assembly. The assembled flight units meet their mission requirements. Measurement data before, after and during environmental testing indicates that the flight units can survive launch and vacuum environment. All flight units have already been mechanically integrated on the ATLAS instrument and thermal hardware have been successfully tested. Optical testing will be demonstarted at the instrument level upon integration of the flight lasers.

\section{REFERENCES}

[1] ICESat-2 Instrument (2014): http://icesat.gsfc.nasa.gov/icesat2/instrument.php

[2] Yu A.W. et al. "Space Laser Transmitter Development for ICESat-2 Mission ", Proc. SPIE 7578, 757809, (2010).

[3] Sawruk N. W. et al. "Space Qualified Laser Transmitter for the NASA ICESat-2 Mission", Proc. SPIE 8599, 85990 (2013). (2001). 